\title{
Viscous Fingering of Non-Newtonian Fluids in a Rectangular Hele-Shaw Cell
}

\author{
Takehiro Yamamoto, Hiroo Kamikawa, Hiromitsu TanaKa, Kiyoji NaKamuRA, and Noriyasu Mori \\ Department of Mechanophysics Engineering, Graduate School of Engineering, Osaka University, \\ 2-1, Yamadaoka, Suita, Osaka 565-0871, Japan \\ (Received: January 11, 2001)
}

\begin{abstract}
Non-Newtonian viscous fingering in a rectangular Hele-Shaw cell was studied. Compressed air was injected into the cell filled with a more viscous fluid. For the more viscous fluids, glycerin was used as a Newtonian fluid, and aqueous solutions of carboxymethylcellulose (CMC) and polyacrylamide (PAA) were used as non-Newtonian fluids. The growth and the structure of viscous fingers were analyzed. The shear-thinning viscosity of the polymer solutions made the 'shielding' effect strong. When the 'shielding' appeared strongly, the fingers formed a branched structure. In the PAA solution, the change in the finger pattern, a sudden growth of thin fingers from a wide finger-tip, was observed. It was supposed that stretch-thickening elongational viscosity and the shear-thinning viscosity were related to this phenomenon.
\end{abstract}

Key Words: Viscous finger / Non-Newtonian fluid / Hele-Shaw cell / Interface / Polymer solution

\section{INTRODUCTION}

Viscous fingers are observed when a less viscous fluid replaces a more viscous fluid in a Hele-Shaw cell; Hele-Shaw cell is a pair of plates separated by narrow gap. The interface between two fluid grows to form finger-like patterns repeating three typical processes, 'spreading', 'splitting', and 'shielding'. Viscous fingering is an interesting phenomenon and has been studied in physics, chemistry, engineering, and many other fields. ${ }^{1,2)}$ Viscous fingering in porous media is one of the Laplace growth phenomena and sometimes shows patterns similar to those observed in the diffusion limited aggregation, DLA and besides, has been studied in connection with oil recovery. ${ }^{1)}$ Furthermore, the viscous fingers form fractal structures under some conditions. ${ }^{2), 3)}$ Nittmann et al. ${ }^{4)}$ replaced an aqueous polymer solution in a rectangular Hele-Shaw cell by water. These fluids are miscible, thus the interfacial tension between the fluids is negligible. They analyzed the structure of a viscous finger using the concept of fractals and showed that the fingering patterns have fractal character. Damme et al.5) used a clay suspension in water as a more viscous fluid and pure water as a less viscous fluid, and analyzed the fractal dimension of the viscous fingers. Buka et al. ${ }^{6)}$ carried out experiments using nematic liquid crystals as a more viscous fluid, and showed that the fingering pattern differed from that of isotropic liquids.
Recently, many workers focused on the viscous fingering in non-Newtonian fluids and investigated the effect of nonNewtonian properties on the finger growth (e.g. Refs.7-16). In plastic industry, non-Newtonian viscous fingers are observed in the polymer processing such as the gas-assisted injection molding and affect the quality of products. Hence it is important to understand the mechanism of the growth of the viscous fingers. Moreover, it is also significant to study the relationship between the finger growth and the non-Newtonian property of polymeric liquids. We studied the effect of nonNewtonian property on the fractal structure of viscous fingers using some aqueous polymer solutions as a more viscous fluid. ${ }^{12,13)}$ As a result, we found that the shear-thinning viscosity of polymer solutions affected the fingering pattern, and the stretch-thickening elongational property of polymer solutions stabilized the interface of the low and high viscous fluids. Wilson ${ }^{14)}$ discussed the Saffman-Taylor instability problem of non-Newtonian viscous fingering. He considered the stability of the fingers in a Hele-Shaw cell filled with the Oldroyd-B fluid or the Ostwald-de Waele power-law fluid, and concluded that the shear-thinning viscosity did not show any interesting effects. On the other hand, recent experimental studies indicated the effect of shear-thinning viscosity on the finger growth (e.g. Ref. 9, 11, 15, and 16). In spite of many recent studies, the mechanics of non-Newtonian viscous fingering has not been well elucidated and is still a challenging topic. 
Table I Interfacial tension $\sigma$ of the test fluids at $20^{\circ} \mathrm{C}$

\begin{tabular}{l|c|c|c|c|c} 
Fluid & glycerin & CMC $1.0 \mathrm{wt} \%$ & CMC 2.0wt $\%$ & PAA $1.0 \mathrm{wt} \%$ & PAA 2.0 wt $\%$ \\
\hline$\sigma[\mathrm{mN} / \mathrm{m}]$ & 63.2 & 75.3 & 75.2 & 76.3 & 75.4
\end{tabular}

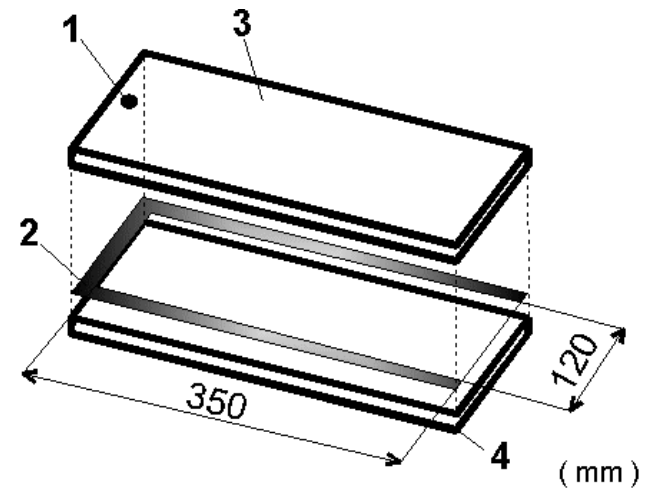

Fig.1 Schematic diagram of a rectangular Hele-Shaw cell: (1) air inlet, (2) spacer (0.2 mm in thickness), (3) upper plate, and (4) lower plate

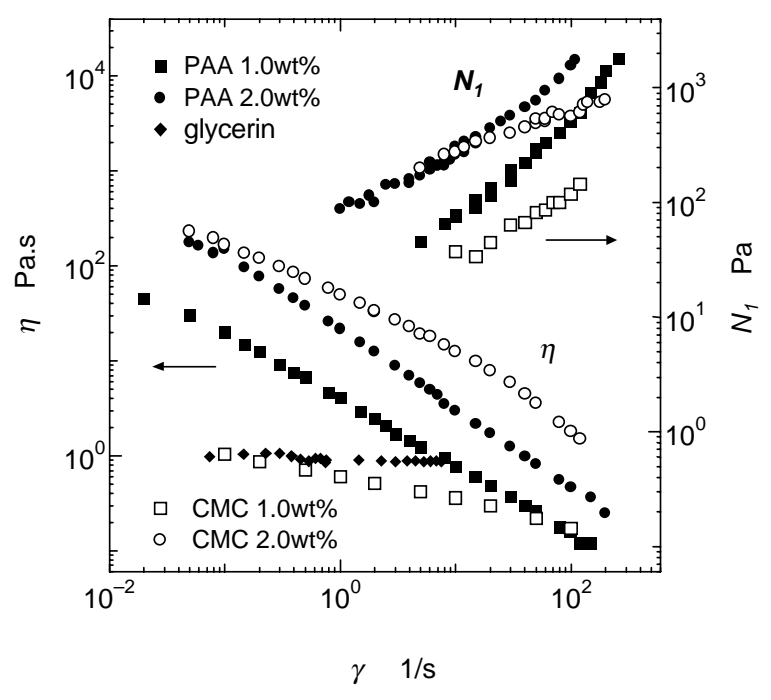

Fig.2 Steady shear viscosity $\eta$ and the first normal stress difference $N_{1}$ of the test fluids versus shear rate $\dot{\gamma}$.

In the present experiment, we used two polymer solutions as the more viscous fluid and investigated the effect of nonNewtonian properties on the fingering pattern and the finger growth in a rectangular Hele-Shaw cell.

\section{EXPERIMENTS}

\subsection{Experimental Set-up and Method}

Fig. 1 shows the schematic diagram of a rectangular HeleShaw cell used in the present study. The cell consisted of a pair of parallel plates, which were separated by a narrow gap. The size of the cell was $350 \mathrm{~mm}$ in length and $120 \mathrm{~mm}$ in width. The plates were $20 \mathrm{~mm}$ in thickness and were fixed by 19 pairs of bolts and nuts for preventing the bending. The gap was kept
$0.2 \mathrm{~mm}$ by a spacer of a plastic sheet.

The cell was filled with a high viscous fluid and compressed air was used as a low viscous fluid. Air compressed by a compressor was injected into the cell at constant pressure; the injection pressure $p$ was adjusted by a precise regulator. We carried out the experiments at various injection pressure.

The growth of the fingers was recorded by a CCD video camera (Panasonic NV-X100). The images were captured into a computer through a video capture board (Canopus Power Movie MP/V) and were transformed into binary images. We analyzed the structure and the growth of the fingers based on the binary images.

\subsection{Test Fluids}

Test fluids used as the high viscous fluid were glycerin, 1.0 and $2.0 \mathrm{wt} \%$ aqueous solutions of carboxymethylcellulose (CMC, Daicel Chemical Industries CMC DAICEL 2260, $M$ $\left.=2.2 \times 10^{5}\right)$, and 1.0 and $2.0 \mathrm{wt} \%$ aqueous solutions of polyacrylamide (PAA, Sanyo Chemical Industries SANFLOC AH70P, $M=1.0 \times 10^{7}$ ). Glycerin is a Newtonian fluid and the other fluids are viscoelastic fluids, thus they are nonNewtonian.

Fig. 2 shows the steady shear viscosity and the first normal stress difference of the test fluids. They were measured with a cone-plate type rheometer (Nippon Rheology Equipment NRM-2000S-NF). This figure shows that glycerin has a constant viscosity and the other fluids have shear-thinning viscosity. The PAA and CMC solutions have elastic property because they show non-zero first normal stress difference. Moreover, the aqueous solution ofPAA has strong stretchthickening property. ${ }^{17), 18)}$ On the other hand, the aqueous solution of CMC has no thread-forming ability and its stretchthickening property is weak.

The surface tension of the test fluids measured with a Du Nouy type tensionmeter are summarized in Table I. The surface tensions of the polymer solutions are almost equivalent and vary within about $1.5 \%$.

\section{RESULTS AND DISCUSSION}

\subsection{Fingering Patterns}

Figs. 3-8 show the binary images of the fingers; the air region and the high-viscous-fluid region were represented by 
white and black pixels, respectively. The patterns when the fingers reach near the end of the cell are shown in Figs.3-7. Fig. 8 shows the patterns at five steps of the finger growth. In these figures, $t$ means time after the onset of the finger growth.

Fig. 3 shows the results for glycerin. The fingers are relatively wide and there are not many branches at low injection pressure ( $p=10 \mathrm{kPa}$ ). The number of the branches increases with increasing the pressure. The 'shielding' effect does not appear clearly, thus the fingers uniformly grow and form high dense structures at every injection pressure. In this case, the finger pattern does not have fractal structure. ${ }^{12)}$

Figs. 4 and 5 show the results for the 1.0 and $2.0 \mathrm{wt} \%$ aqueous solutions of CMC, respectively. Because viscosity of the $2.0 \mathrm{wt} \%$ solution is higher than that of the $1.0 \mathrm{wt} \%$ solution, the speed of the finger growth is slower in the case of the $2.0 \mathrm{wt} \%$ solution. Similarly to the case of glycerin at low injection pressure, the fingers in the $1.0 \mathrm{wt} \% \mathrm{CMC}$ solution are
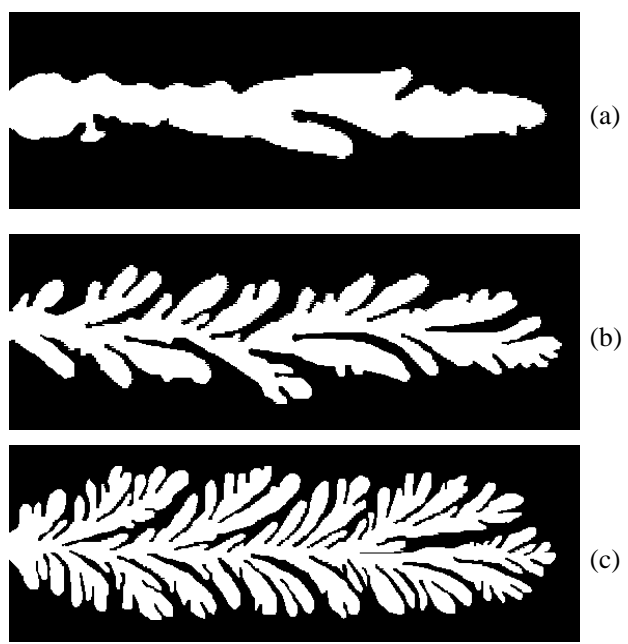

Fig.3 Fingering patterns in glycerin. (a) $p=10 \mathrm{kPa}, t=242 \mathrm{~s}$, (b) $p=20 \mathrm{kPa}$, $t=24.0 \mathrm{~s}$, and (c) $p=30 \mathrm{kPa}, t=6.10 \mathrm{~s}$.

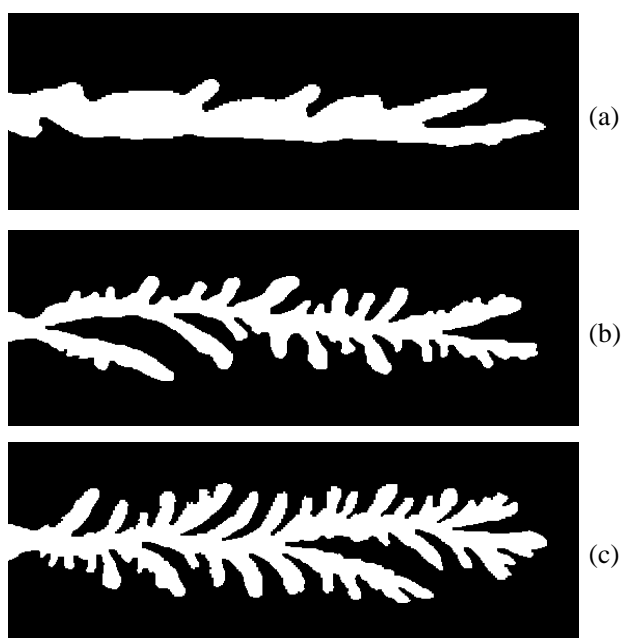

Fig.4 Fingering pattern in the CMC $1.0 \mathrm{wt} \%$ solution. (a) $p=10 \mathrm{kPa}$, $t=1161 \mathrm{~s}$, (b) $p=20 \mathrm{kPa}, t=7.50 \mathrm{~s}$, and (c) $p=30 \mathrm{kPa}, t=1.22 \mathrm{~s}$.
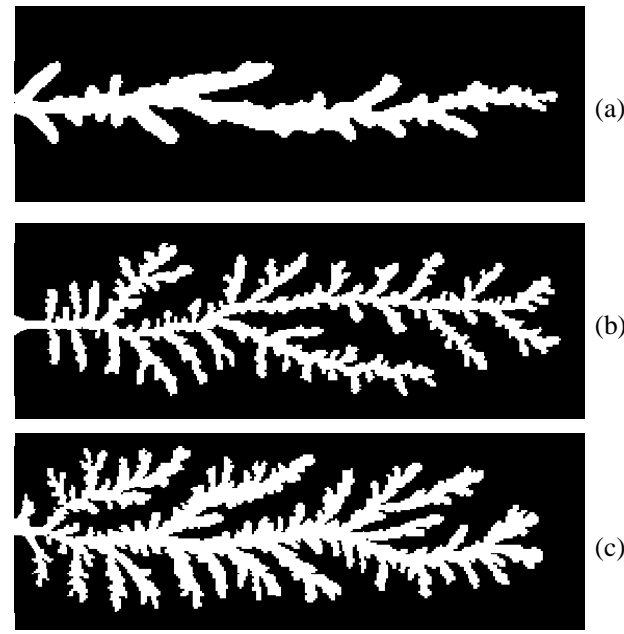

Fig.5 Fingering pattern in the CMC $2.0 \mathrm{wt} \%$ solution. (a) $p=20 \mathrm{kPa}$, $t=1506 \mathrm{~s}$, (b) $p=30 \mathrm{kPa}, t=27.4 \mathrm{~s}$, and (c) $p=40 \mathrm{kPa}, t=2.75 \mathrm{~s}$.
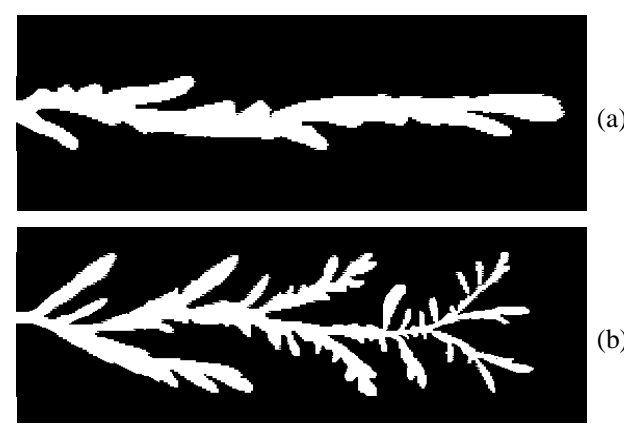

Fig.6 Fingering pattern in the PAA $1.0 \mathrm{wt} \%$ solution. (a) $p=10 \mathrm{kPa}$, $t=49.2 \mathrm{~s}$ and (b) $p=20 \mathrm{kPa}, t=1.60 \mathrm{~s}$.
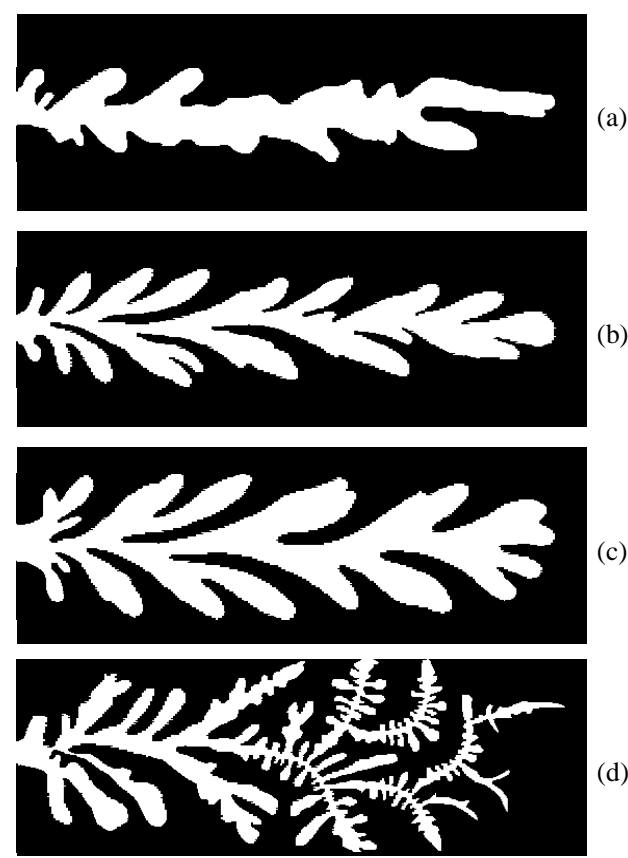

Fig.7 Fingering pattern in the PAA $2.0 \mathrm{wt} \%$ solution. (a) $p=10 \mathrm{kPa}$, $t=2257 \mathrm{~s}$, (b) $p=20 \mathrm{kPa}, t=27.7 \mathrm{~s}$, (c) $p=30 \mathrm{kPa}, t=9.00 \mathrm{~s}$, and (d) $p=40 \mathrm{kPa}, t=1.67 \mathrm{~s}$. 

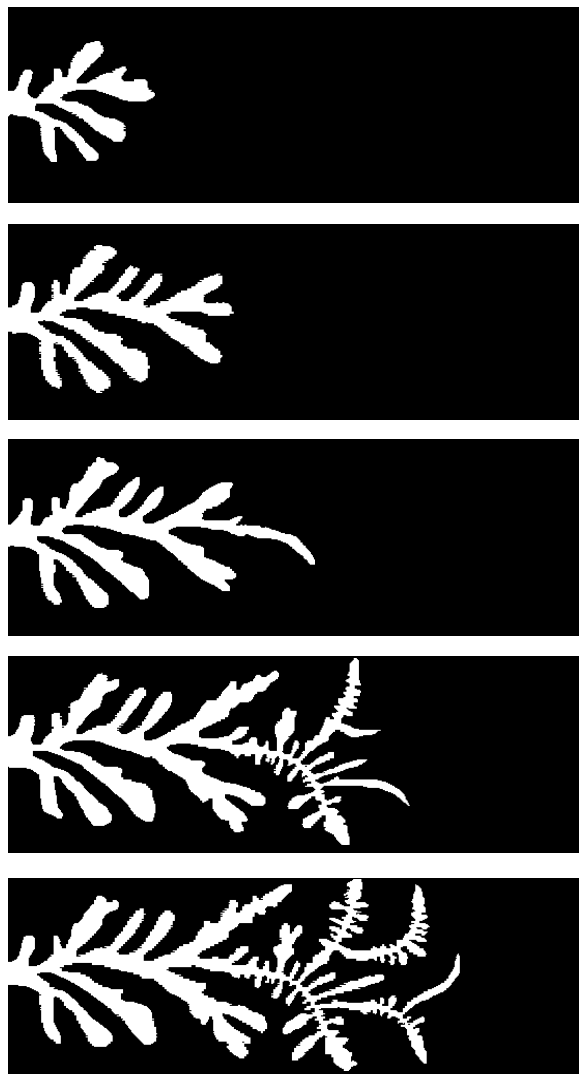

Fig.8 Finger growth of the PAA $2.0 \mathrm{wt} \%$ solution at $p=40 \mathrm{kPa}$ at $t$ $=0.80,1.25,1.32,1.52$, and $1.60 \mathrm{~s}$ (from top to bottom).

wide and there are not many branches. In other cases, however, thin fingers are extended and form a branched structure. The branched structure is attributed to the strong effect of 'shielding', which are intensified by the non-uniformity of viscosity near the finger-tip due to the shear-thinning property. Sader et al. ${ }^{15)}$ have pointed out that the shear-thinning viscosity played an important role in fractal growth.

Fig. 6 and 7 show the results for the 1.0 and $2.0 \mathrm{wt} \%$ aqueous solutions of PAA. In addition, the growth of the fingers in the $2.0 \mathrm{wt} \%$ PAA solution is shown in Fig.8. The fingers are wide and the shielding effect is weak at the low injection pressure. The curvature of the finger-tip of thePAA solutions is larger than that of the CMC solutions. At the high injection pressure, however, the fingers change the structure during their growth: Thin fingers suddenly grow from a wide finger-tip and form a highly branched structure (Fig.8). The similar phenomenon was also observed in the experiment of viscous fingering in the PAA solutions in a radial Hele-Shaw cell. $^{12,13)}$

The injection pressure was relatively high compared with previous studies of viscous fingering. The structural change has not been observed at low injection pressure, thus large change in pressure gradient near the finger-tip was a cause of this phenomenon. In addition, this structural change was not

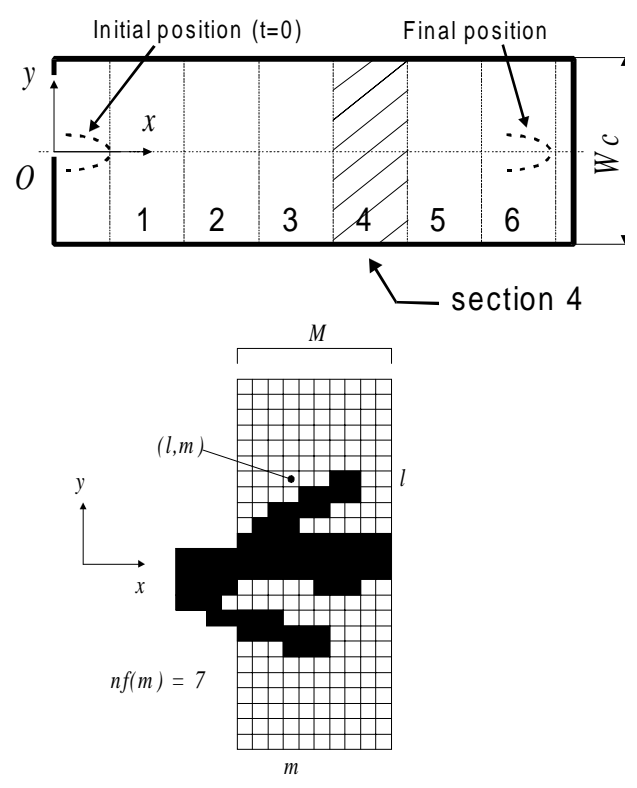

Fig.9 Section for the analysis (upper) and image of pixels in one section (lower).

observed in the case of the CMC solutions of any concentrations used in the present study. Since the CMC solutions and the PAA solutions are similar in shear property but are much different in elongational property, we supposed that the change in finger patterns was related to the elongational property of the test fluids. We here propose a mechanism of the structural change as follows: In general, when tension between two fluids is strong, the interface is stable, and wide fingers grow. Because of the stretchthickening elongational viscosity, apparent interfacial tension becomes strong and then the interface spreads and the wide fingers grow. The curvature of the finger-tip becomes relatively large. However, after the break down of the interfacial stability, thin fingers begin to grow and form a branched structure because of the shielding effect intensified by the shearthinning property. Further study will need to clarify the mechanism and especially, the quantitative evaluation of the elongational property of polymer solutions is required. However, the measurement of elongational viscosity of polymer solution itself is still a challenging issue in rheology.

\subsection{Analysis}

To analyze quantitatively the finger growth, we divided the cell into six regions as shown in Fig.9 and evaluated four values, $N_{f}, W_{f}, V_{f}$, and $Y_{f}$ in each section based on the binary images. These values are defined as follows: $N_{f}$ is mean number of finger branches in a section and is calculated by

$$
N_{f}=\sum_{m} n_{f}(m) / M
$$


where $M$ is total number of the row in the section and $n_{f}(m)$ is number of branches in the $m$ th row (see Fig.9). $W_{f}$ and $V_{f}$ are mean branch width and mean velocity of the finger-tip, respectively. These values were evaluated based on the binary digital images. In addition, $Y$ is a quantity for evaluating the spread of the finger branches in the $y$-direction and is defined by

$$
Y=\sqrt{\int_{A} y^{2} d A / A},
$$

where $y$ indicates the $y$-coordinate of a point in the air region. $A$ is the total area of the air region. The term $\int_{A} y^{2} d A$ is the moment of inertia around the $x$-axis. For the binary images, we used the following equation:

$$
Y=\sqrt{\sum_{i} y_{i}^{2} / n}
$$

where $y_{i}$ indicates the distance of the $i$ th pixel from the center of the cell. $W_{f}, V_{f}$, and $Y_{f}$ were normalized as follows: $W_{f}^{*}=W_{f}$ $/ W_{c}, V_{f}^{*}=V_{f} T / W_{c}$, and $Y^{*}=Y / W_{c}$, where $W_{c}$ is the cell width. $T$ is the characteristic time and was set to $1 \mathrm{~s}$ in the present study. In the following discussion, these non-dimensional values are used.

Fig. 10 shows the dependence of $N_{f}^{*}$ and $W_{f}^{*}$ on $V_{f}^{*}$. The finger-tip velocity is faster at higher injection pressure and in the region nearer the exit of the cell where the pressure gradient is large.

As shown in the results of glycerin, with increasing $V_{f}^{*}, N_{f}^{*}$ increases and $W_{f}^{*}$ decreases; $W_{f}^{*}$ does not vary so much when $V_{f}^{*}$ is large. In the cases of both the CMC and the PAA solutions, qualitatively the same tendency is observed in low velocity region. In high velocity region, however, $N_{f}^{*}$ does not differ with $V_{f}^{*}$. This fact means the decrease of number of the fingers due to the shielding effect.

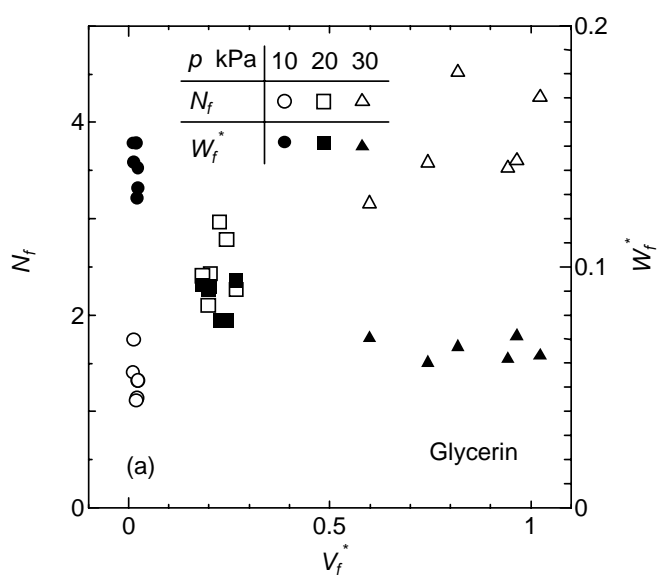

Fig.10 (continued)
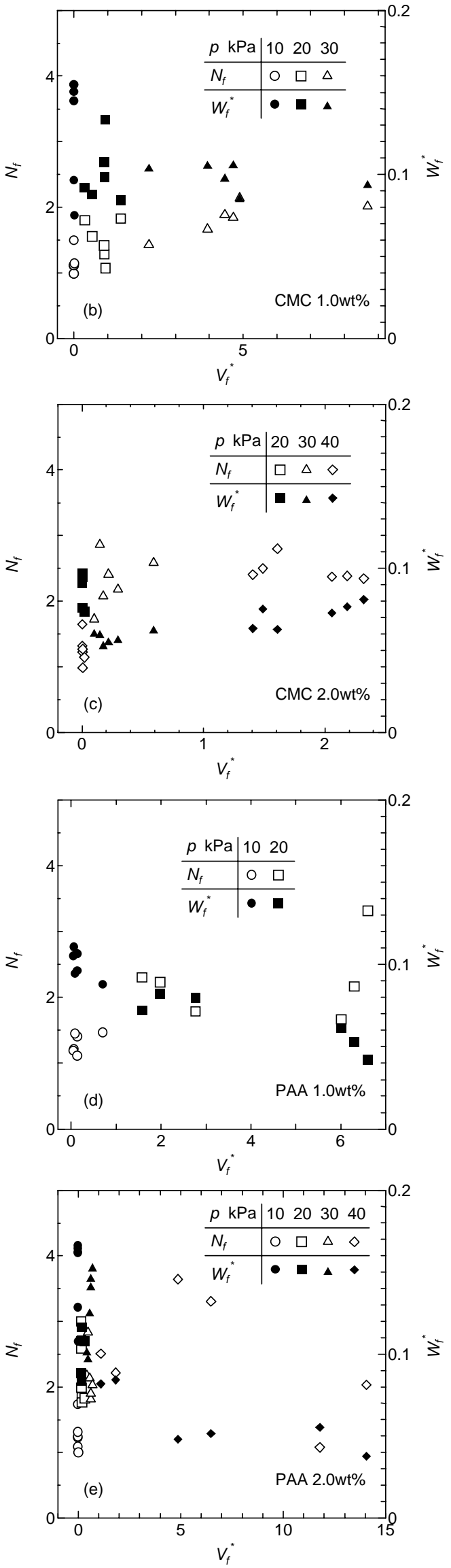

Fig.10 Dependence of $N_{f}^{*}$ and $W_{f}^{*}$ on $V_{f}^{*}$. (a) glycerin, (b) CMC 1.0wt $\%$ solution, (c) CMC $2.0 \mathrm{wt} \%$ solution, (d) PAA $1.0 \mathrm{wt} \%$ solution, and (e) PAA $2.0 \mathrm{wt} \%$ solution. 

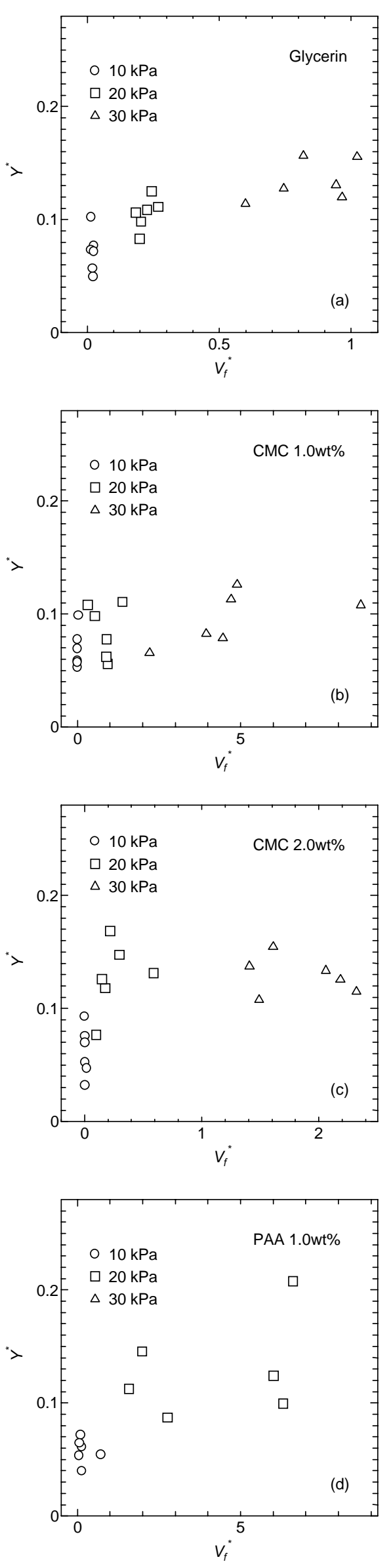

Fig.11 (continued)

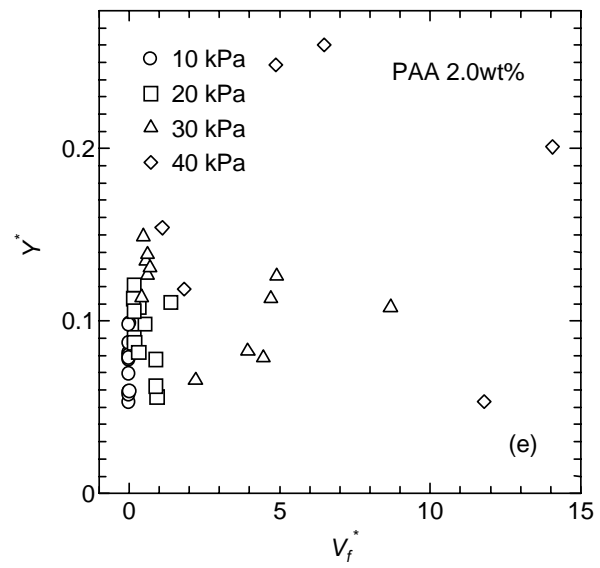

Fig.11 Dependence of $Y_{f}^{*}$ on $V_{f}^{*}$. (a) glycerin, (b) CMC $1.0 \mathrm{wt} \%$ solution, (c) CMC $2.0 \mathrm{wt} \%$ solution, (d) PAA $1.0 \mathrm{wt} \%$ solution, and (e) PAA $2.0 \mathrm{wt} \%$ solution.

In the case of the PAA solutions, $N_{f}^{*}$ suddenly decreases when $V_{f}^{*}$ is very large because the shielding effect is strong after the structural change. In this region $W_{f}^{*}$ decreases. This result indicates a few thin fingers grow after the structural change. In the result for the $2.0 \mathrm{wt} \%$ PAA solution at $p=40$ $\mathrm{kPa}$, thin fingers begin to grow during the growth, thus $V_{f}^{*}$ reaches very large value.

Fig. 11 shows the dependence of $Y^{*}$ on $V_{f}^{*}$. In general, when the tip speed is high, the splitting of the finger-tip is accelerated thus the finger branches notably. When $V_{f}^{*}$ is relatively small, $Y_{f}^{*}$ tends to increase with increasing $V_{f}^{*}$. When $V_{f}^{*}$ is large, $Y^{*}$ changes little for the CMC solutions, while $Y^{*}$ suddenly increases for the PAA solutions. As shown in the results of the $1.0 \mathrm{wt} \%$ PAA solution at $p=20 \mathrm{kPa}$ and of the $2.0 \mathrm{wt} \%$ PAA solution at $p=40 \mathrm{kPa}$, the finger pattern changes and $Y^{*}$ suddenly increases when the finger-tip speed is high. The sudden change in $Y^{*}$ corresponds to this phenomenon.

\section{CONCLUSION}

Non-Newtonian viscous fingering in the rectangular HeleShaw cell for relatively high injection pressure was studied. The fingers in glycerin formed dense structures, while the fingers in non-Newtonian fluids formed branched structures. It was found that the shear-thinning viscosity intensified the shielding effect, thus the fingering pattern becomes the branched shape. Moreover, the fingering pattern in the PAA solutions changed during the growth and this phenomenon was unique to the experiments at high injection pressure. The origin of this phenomenon was supposed to be the shearthinning and stretch-thickening properties. 


\section{REFERENCES}

1) Homsy, GM, Annu Rev Fluid Mech, 19, 271 (1987).

2) Vicsek T, “Fractal Growth Phenomena”, (1989) World Scientific Publishing, Singapore.

3) Feder J, “FRACTALS”, (1988) Plenum Press, New York.

4) Nittmann J, Daccord G, Stanley HE, Nature, 314, 141 (1985).

5) Damme HV, Obrecht F, Levitz P, Gatineau L, Laroche C, Nature, 320,731 (1986).

6) Buka A, Kertesz J, Vicsek T, Nature, 323, 424 (1986).

7) Bonn D, Meunier J, Phys Rev Lett, 79, 2662 (1997).

8) Poire EC, Amar MB, Phys Rev Lett, 81, 2048 (1998).

9) Kawaguchi M, Shibata A, Shimomoto K, Kato T, Phys Rev E, 58, 785 (1998).
10) Kondic L, Shelley MJ, Palffy-Muhoray P, Phys Rev Lett, 80, 1433 (1998).

11) Amar MB, Poire EC, Phys Fluids, 11, 1757 (1999).

12) Yamamoto $\mathrm{T}$, Kamikawa $\mathrm{H}$, Nakamura $\mathrm{K}$, Nihon Reoroji Gakkaishi, 24, 105 (1996) (in Japanese).

13) Yamamoto T, Kamikawa H, Nakamura K, Proc 2nd Pacific Rim Conf on Rheology, 309 (1997).

14) Wilson SDR, J Fluid Mech, 220, 413 (1990).

15) Sader JE, Chan DYC, Hughes BD, Phys Rev E, 49, 420 (1994).

16) Weisser EM, Boger DV, Proc 2nd Pacific Rim Conf on Rheology, 297 (1997).

17) Fuller GG, Cathey CA, Hurbbard B, Zebrowski BE, J Rheol, 31, 235 (1987)

18) Koshiba T, Mori N, Nakamura K, Trans Jpn Soc Mech Eng, 62 B, 3890 (1996) (in Japanese) 